P8 (continued)

moderate or mild without example activities, all students reported that the listed example activities aided comprehension. Chronological understanding was strong with $96 \%$ providing sound responses for the difference between 30 and 60 minutes and $78 \%$ for the difference between 30 days and 6 months. The mixed horizontal-vertical layout was navigated well by $92 \%$.

Conclusions and Implications: Fuel for Fun PA items demonstrated face validity and utility to assess PA interventions with 4 th grade youth. However, comprehension may be improved further by simplifying specific terms and changing layout to a vertical-only format.

Funding: USDA, Rochester Institute of Technology

\section{P9 Consumption of Snacks and Carbonated Beverages are Higher in ADHD Korean Children Aged 7-10 Years}

Seong Sook Park, MS, Shin-Seo Elementary School; Hyun-Kyoung Bang, MS, Keimyung University; Mi-Ja Choi, choimj@kmu.ac.kr, Keimyung University, 42601, Daegu, Dalseo-gu, Korea 42601

Objective: Attention-Deficit Hyperactivity Disorder (ADHD) is common in children. But little is known about the dietary behaviors of Korean children with ADHD. The objective was to examine whether the number of snacks, type of snack, and attitude of mothers was associated with dietary behaviors of children with ADHD.

Methods: A total of 223 school children aged 7-10 yearsold and their mothers were enrolled in Daegu City, Korea. Parents completed a socioeconomic status and were asked questions, such as frequency of children's snacking, kinds of snack, reasons for snacks, and preparation of snack. Teachers were informed of the study and requested to cooperate. The children were screened ADHD by using Korean ADHD Rating Scale by their school teachers.

Outcome Measures and Analysis: Data were analyzed using SAS version 9.3 (SAS Institute, Inc.). Significant differences were defined as $\mathrm{p}<0.05$. Student's t-test were used to determine significant differences between groups. Results: Both the control and ADHD group were all snacking. Education levels were low, and employment level is high. Mothers of the ADHD group are more likely to provide snacks to children based on children's preference for food, such as carbonated beverage, processed food, and confectionery, compared to the mothers of the control group.

Conclusion: The present study revealed increased consumption of confectionery snacks and carbonated beverages in ADHD children.

Funding: None

\section{P10 Differences in Overall Diet Quality in US Children Who Consumed or Skipped Breakfast by Weight Status}

Lillian E. Delatore, The Ohio State University; Colleen K. Spees, PhD, MEd, RDN, LD, FAND;
Christopher A. Taylor; PhD, RDN, LD, FAND, taylor.1043@ osu.edu, The Ohio State University, 453 West 10th Avenue, 306 Atwell Hall, Columbus, OH 43210

Objective: Breakfast consumption has been linked to numerous positive effects in children related to improved dietary quality and obesity prevention. The purpose of this study was to assess differences in dietary patterns between children who consumed breakfast versus those that do not by weight status.

Study Design, Setting, Participants: Dietary intakes of 13- to 18-year-old children $(n=4,110)$ from the 20052012 National Health and Nutrition Examination Survey and were categorized by breakfast consumption.

Outcome Measures and Analysis: Dietary intakes were assessed using a 24-hour recall. Breakfast consumption was tallied using self-reported meal identification for foods reported. Diet quality was tabulated using the Healthy Eating Index-2010 score (HEI-2010, 0-100 range). Children's weight status was categorized using BMI-forage percentiles into normal weight, overweight, and obese classifications.

Results: Approximately $72 \%(n=2,870)$ of children ate breakfast on the day of report. Normal weight children were more likely to eat breakfast (74\%) than overweight and obese children (66\%, respectively) on the day of intake. Total diet quality was better in children who ate breakfast (HEI-2010: 42.1-43.0) than those who skipped breakfast (HEI-2010: 37.8-39.5) with little differences in HEI-2010 noted by weight status. Children who ate breakfast had better diet quality scores for fruit, whole fruit, whole grains, dairy products, and empty calories, but had lower scores for vegetables, protein foods, fatty acids, and refined grains.

Conclusions and Implications: Overall diet quality was better in children who ate breakfast than those that did not, regardless of weight status. Breakfast consumption is related to better dietary patterns and further research is needed to explore the drivers of the intakes that create those differences.

Funding: None

\section{P11 Eat Smart in Parks: Impacts of a Youth Photovoice Project}

Sonja Wilhelm Stanis,PhD, sonjaws@missouri.edu, University of Missouri, 105 ABNR Building, MO 65211; Kimbery Keller, PhD, CFLE, University of Missouri; Cindy DeBlauw, LDN, RD; Natalie Hampton, $M P H$

Objective: Interest in healthy food and beverage options in parks, recreation centers and public spaces is growing. As part of Eat Smart in Parks (ESIP)-a nutrition environment initiative named a promising practice by SNAP-Ed-a youth photovoice engagement project launched in 2015 to help address the nutrition issues and needs identified by youth in Missouri. This study examined if participating in this photovoice project changed youth's perceptions regarding parks and healthy food environments.

Continued on page S27 